\title{
Yielding of weakly attractive nanoparticle networks $\dagger$
}

\author{
André R. Studart, ${ }^{* a}$ Esther Amstad ${ }^{b}$ and Ludwig J. Gauckler ${ }^{b}$ \\ Received 5th April 2011, Accepted 18th May 2011 \\ DOI: 10.1039/c1sm05598d
}

We propose a scaling law that explicitly correlates the macroscopic yield stress of weakly attractive nanoparticle networks with features of the microscopic aggregates that form the percolating gel. The proposed law correctly predicts experimental data obtained from a model colloidal system covering a wide range of particle sizes, volume fractions and interparticle attractive forces. The agreement between theory and experiments supports the idea that yielding is ultimately caused by the rupture of a few interparticle bonds within the aggregates of the mechanically loaded network.

Colloids consisting of weakly attractive nanoparticles are important in many technological processes and products in areas ranging from printing, to cosmetics, to pharmaceutics, to food and materials processing. ${ }^{1-5}$ Weak attractive interactions between nanoparticles lead to colloidal networks exhibiting solid-like, elastic behavior if the particle volume fraction is higher than the percolation threshold. Shearing of these colloidal systems ruptures the solid-like particle network and thus drastically changes its rheological response to a fluid-like behavior. The transition between these very distinct rheological states is characterized by a finite yield stress $\left(\tau_{\mathrm{y}}\right)$, which is defined by the shear stress needed to fluidize an initially elastic particle network.

Since macroscopic yielding of these networks originates from the microscopic van der Waals attractive forces between particles, many theoretical and experimental attempts have been made to correlate the yield stress of colloidal networks with the magnitude of interparticle forces, $F_{\max }$ (or interparticle potential, $U_{\mathrm{T}}$ ), the particle radius, $a$, and volume fraction, $\phi .^{6-14}$ According to most microrheological models, $\tau_{\mathrm{y}}$ scales as follows: ${ }^{6,7,15-18}$

$$
\tau_{\mathrm{y}} \propto \frac{F_{\max }^{\alpha} \phi^{\gamma}}{a^{\beta}},
$$

where $\alpha=1$ and $\beta$ and $\gamma$ are power exponents ranging from 1.5 to $2.0^{\mathbf{8 , 1 0 , 1 3 , 1 9 - 2 2}}$ and from 1.4 to $5.5^{10-14,19-26}$ respectively. $\gamma$ is usually correlated with the microstructure of the colloidal particle network through the fractal dimension of its aggregates, $d_{\mathrm{f}}$.

Although the above scaling law has successfully described experimental data in specific colloidal systems, little agreement is obtained

${ }^{a}$ Complex Materials, Department of Materials, ETH Zurich, 8093 Zurich, Switzerland.E-mail: andre.studart@mat.ethz.ch

${ }^{b}$ Nonmetallic Inorganic Materials, Department of Materials, ETH Zurich, 8093 Zurich, Switzerland

$\uparrow$ Electronic supplementary information (ESI) available. See DOI: $10.1039 / \mathrm{c} 1 \mathrm{sm} 05598 \mathrm{~d}$ for the values of the power exponents $\beta$ and $\gamma$. Moreover, information on the actual mechanisms that determine yielding at the particle level is scarce.

In this communication, we propose and experimentally verify a simple scaling law that predicts the yield stress of weakly attractive colloidal gels and suggests that the yielding process is governed by the rupture of a few interparticle bonds within the aggregates.

We propose that the yield stress of attractive nanoparticle networks can be described by the simple explicit relation:

$$
\tau_{\mathrm{y}} \approx \frac{\phi_{\mathrm{eff}}^{2 /\left(3-d_{\mathrm{f}}\right)} F_{\max } \sum_{i=1}^{j} \cos \theta_{i}}{\pi a^{2}},
$$

where $\phi_{\mathrm{eff}}$ is the effective volume fraction of particles, $j$ is the number of critical load-bearing interparticle bonds that have to be ruptured within each aggregate to cause macroscopic yielding and $\theta_{i}$ is the angle between the loading and bonding directions for the interparticle bond $i$ (Fig. 1a). We experimentally find that $d_{\mathrm{f}}=2.36$ and $0.7<\sum_{i=1}^{j} \cos \theta_{i}<2$ for the weakly attractive concentrated networks investigated here.

To theoretically derive this expression we hypothesize that the network is formed by tightly packed fractal aggregates, each of which is subjected to the external force $F_{\text {ext }}$, as schematically shown in Fig. 1a. We assume that macroscopic yielding of the gel is caused by the rupture of the weakest links within such aggregates. Considering each aggregate as a representative volume of the entire network structure, the macroscopic yield stress $\tau_{\mathrm{y}}$ can be described by the strength of the aggregate, as follows: ${ }^{.25,27,28}$

$$
\tau_{\mathrm{y}} \approx \frac{F_{\mathrm{ext}}}{\pi \xi^{2}}
$$

where $F_{\text {ext }}$ is the external shear force applied onto each aggregate and $\xi$ is the aggregate radius.

We further hypothesize that the rupture of an aggregate requires break-up of a critical number $j$ of interparticle bonds (weak links) within such aggregate. Upon external loading, the applied force $F_{\text {ext }}$ is counteracted by the sum of all critical microscopic interparticle forces projected along the shear plane (Fig. 1a). Thus, the maximum external force that has to be applied to initiate yielding is given by $F_{\text {ext }} \approx F_{\max } \sum_{i=1}^{j} \cos \theta_{i}$.

Due to the fractal nature and self-similarity of these systems, ${ }^{\mathbf{1 0}}$ the aggregate radius, $\xi$, can be approximated by $a \phi_{\mathrm{int}}{ }^{1 /\left(d_{1}-3\right)}$, where $\phi_{\mathrm{int}}$ is 
a
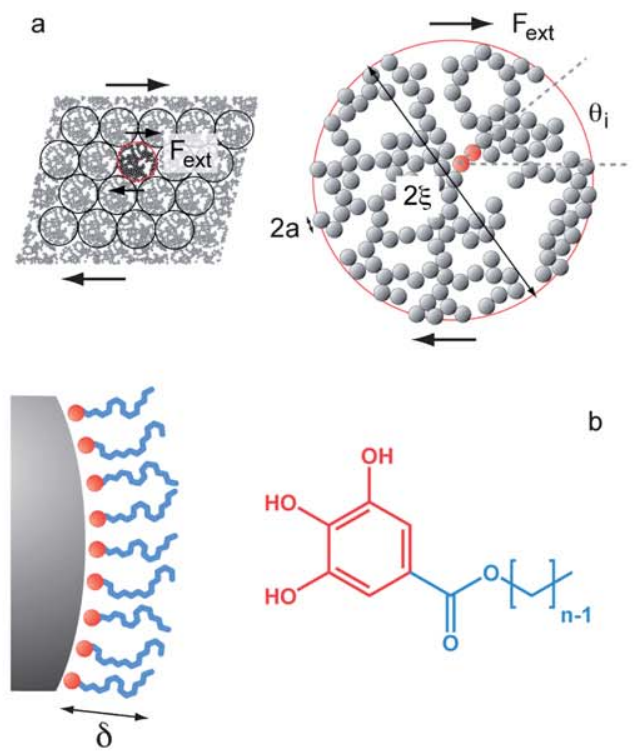

b
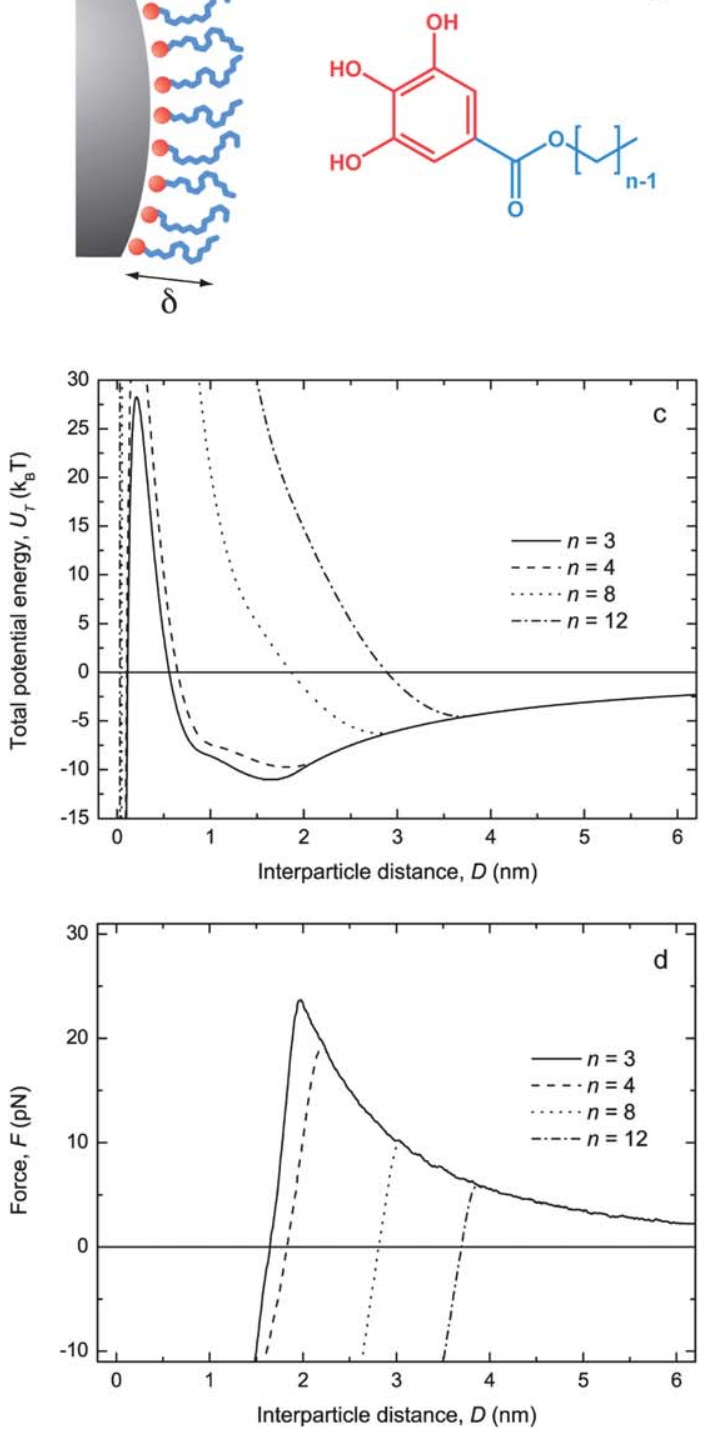

Fig. 1 (a) Schematic drawing of a gel network submitted to simple shear, showing an aggregate within the gel and the critical interparticle bond within such aggregate (assuming $j=1$ ). (b) Tailor-made ligand molecules adsorbed on the particle surface, leading to a well-defined adlayer thickness $\delta$. Examples of calculated (c) total potential energy $U_{\mathrm{T}}$ and (d) total force $F$ between interacting alumina particles coated with ligand molecules containing different number of carbons in the alkyl tail, $n$ (particle size, $2 a=65 \mathrm{~nm}$ ).

the aggregate internal volume fraction. For a volume fraction of aggregates in the network of $\phi_{\mathrm{a}}$, one can obtain $\phi_{\text {int }}=\phi_{\text {eff }} / \phi_{\mathrm{a}}$. If the aggregates are assumed to form an interpenetrating space-filling network, $\phi_{\mathrm{a}} \approx 1$ and thus $\phi_{\mathrm{eff}} \approx \phi_{\mathrm{int}} \cdot{ }^{10}$ Substitution of these relations into eqn (3) leads to the yield stress expression proposed.

To experimentally assess the predictive nature of eqn (2), we thoroughly investigate the rheological behavior of a previously reported colloidal system ${ }^{29}$ that allows for deliberate control of $F_{\max }$, $a$ and $\phi_{\text {eff. }}$ Colloidal networks containing particles with 28, 65 and $116 \mathrm{~nm}$ in diameter and volume fractions, $\phi$, ranging from 0.05 to 0.35 were studied.

The attractive force $F_{\max }$ was manipulated by adsorbing welldefined ligand molecules on the particle surface. Such molecules exhibit the same anchoring group but alkyl chains of different lengths (Fig. 1b). By selecting an anchoring group displaying high affinity to the particle surface and using solvent mixtures that favor an extended conformation of the alkyl chain towards the liquid medium, a densely packed steric layer of well-defined thickness $(\delta)$ in the range of $0.97-1.90 \mathrm{~nm}$ is formed around the particles. Based on our earlier study, ${ }^{29}$ toluene was used as the liquid medium of networks containing molecules with longer hydrocarbon chains $(n \geq 8)$, whereas a mixture of $35 \mathrm{wt} \%$ toluene and $65 \mathrm{wt} \%$ methyl ethyl ketone was taken as the liquid phase of networks containing shorter ligands ( $n=3$ and 4$)$. The effective volume fraction of particles, $\phi_{\mathrm{eff}}$, is calculated from the adlayer thickness, $\delta$, using the relation: $\phi_{\mathrm{eff}}=\phi[1+(\delta / a)]^{3} \cdot{ }^{29}$ A detailed experimental description of this model system and its rheological characterization is provided in ref. 29.

The maximum attractive force, $F_{\max }$, is estimated from the potential energy between interacting particles, $U_{\mathrm{T}}$. The low dielectric constant of the solvents used as liquid media prevents the formation of electrically charged sites on the surface of particles. In the absence of surface charges, the steric layer provided by the surface ligands results in a short-range repulsive energy that counterbalances the attractive van der Waals energy at very short interparticle distances. The repulsive energy arising from the steric layer is caused by a local increase in osmotic pressure and by the elastic compression of the surface adsorbed molecular chains as the particles approach one another.

We calculate the total potential energy between a pair of interacting particles $\left(U_{\mathrm{T}}\right)$ by summing the repulsive steric energy and the attractive van der Waals energy at different interparticle distances (see ESI $\dagger$ ). While the repulsive steric energy dominates at short distances, the long-range character of the van der Waals attractive energy leads to a well-defined secondary minimum in the total potential energy curves, $U_{\mathrm{T}}$, as shown in Fig. 1c.

The force between particles can be obtained from the derivative of the potential energy $U_{\mathrm{T}}$ with respect to the interparticle distance $D$. Such derivation leads to the force curves shown in Fig. 1d. The use of ligands of variable chain lengths enables the formation of attractive potential wells with depths ranging from -1.7 to $-11.0 k T$ and maximum attractive forces varying from 0.47 to $24 \mathrm{pN}$.

The attractive forces between particles result in colloidal networks exhibiting pronounced yielding behavior. This is evidenced by the finite stress needed to rupture the initially elastic network and promote flow of the colloidal system, as indicated in the stress versus strain rate curves shown in Fig. 2.

To determine the yield stress of these colloidal networks under various experimental conditions, we use the Casson equation to fit the rheological data shown in Fig. 2:

$$
\tau^{1 / 2}=\tau_{\mathrm{C}}^{1 / 2}+\left(\eta_{\mathrm{C}} \dot{\gamma}\right)^{1 / 2}
$$



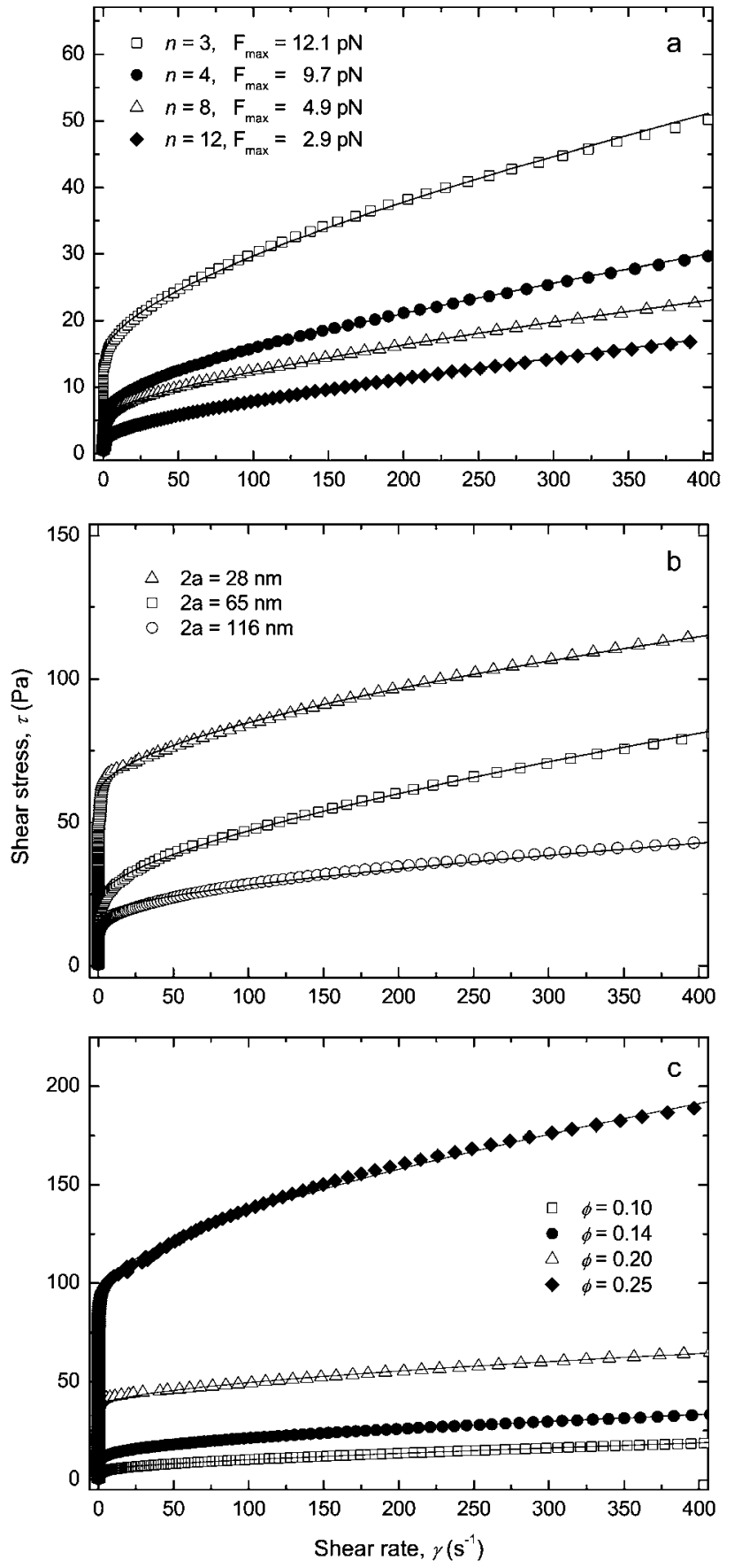

Fig. 2 Representative shear stress versus shear rate curves obtained for colloidal networks containing (a) ligand molecules with different number of carbons $n$ in the alkyl tail $(2 a=65 \mathrm{~nm}, \phi=0.14),{ }^{29}$ (b) particles of different diameters, $2 a(n=4, \phi=0.20)$, and (c) different particle volume fractions, $\phi(2 a=28 \mathrm{~nm}, n=12)$.

where $\tau$ is the shear stress, $\dot{\gamma}$ is the shear rate, $\tau_{\mathrm{y}}$ is the yield stress and $\eta$ is the viscosity.

The Casson relation accurately describes the rheological curves (solid lines in Fig. 2). In a first qualitative analysis, the influence of $F_{\max }, a$ and $\phi$ on $\tau_{\mathrm{y}}$ follows the trends expected from eqn (1). The yield stress, $\tau_{\mathrm{y}}$, increases for increasing $\phi$ and $F_{\max }$, and decreases for larger particle sizes $a$.
Systematic variation of the parameters $F_{\max }, a$ and $\phi_{\text {eff }}$ allows us to experimentally verify the proposed model (eqn (2)). We first analyze the effect of the interparticle force on the rheological response of the network by plotting the particle size-normalized yield stress, $\tau_{\mathrm{y}} a^{2}$, as a function of $F_{\max }$ for a fixed effective volume fraction of 0.2 (Fig. 3a) assuming $\sum_{i=1}^{j} \cos \theta_{i}=1$. In a $\log -\log$ plot, the experimental data can be nicely fit with a linear equation $\left(R^{2}=0.91\right)$, whose slope corresponds to the power exponent $\alpha$ (eqn (1)). Remarkably, a value of 1.004 is obtained from the linear fit to the normalized data (Fig. 3a), experimentally confirming the proposed linear relation between $\tau_{\mathrm{y}}$ and $F_{\max }$ (eqn (2)).

Likewise, the power exponent $\beta$ in eqn (1) is experimentally determined by plotting the force-normalized yield stress, $\tau_{y} / F_{\max }$, as a function of the particle radius, $a$, for a fixed effective volume fraction of 0.2 assuming $\sum_{i=1}^{j} \cos \theta_{i}=1$ (Fig. 3b). In a double logarithm graph, the experimental data can be accurately fit $\left(R^{2}=0.94\right)$ with a linear equation with a slope of 2.02 , which agrees very well with the theoretical value of 2 proposed in eqn (2).

Using $\alpha=1$ and $\beta=2$, one can normalize the yield stress with respect to both the particle size and interparticle force to eventually compare all the experimental data into one single graph displaying $\tau_{\mathrm{y}} a^{2} / F_{\max }$ as a function of the effective volume fraction of particles, $\phi_{\text {eff }}$ (Fig. 4). Remarkably, the initially scattered raw data obtained for
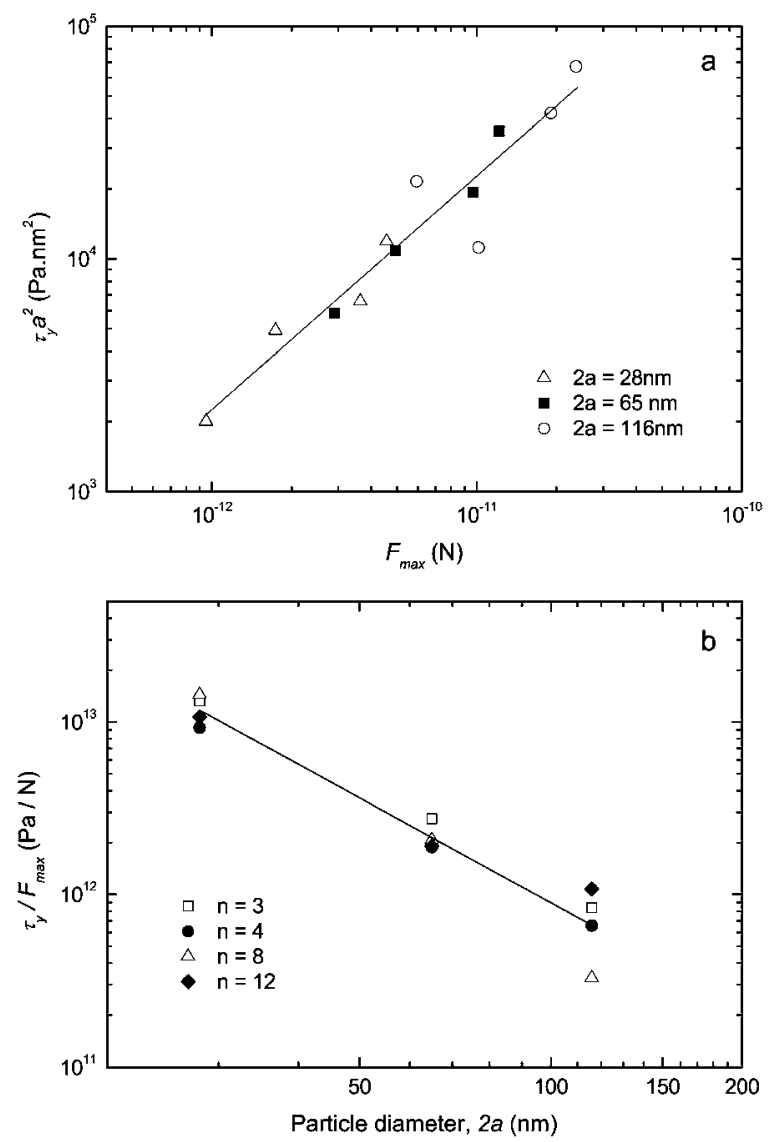

Fig. 3 Effect of the (a) maximum attractive force $F_{\max }$ and (b) particle diameter $2 a$ on the normalized yield stress $\tau_{\mathrm{y}} a^{2}$ and $\tau_{\mathrm{y}} / F_{\max }$, respectively, for an effective volume fraction of particles, $\phi_{\mathrm{eff}}$, of 0.2 . 

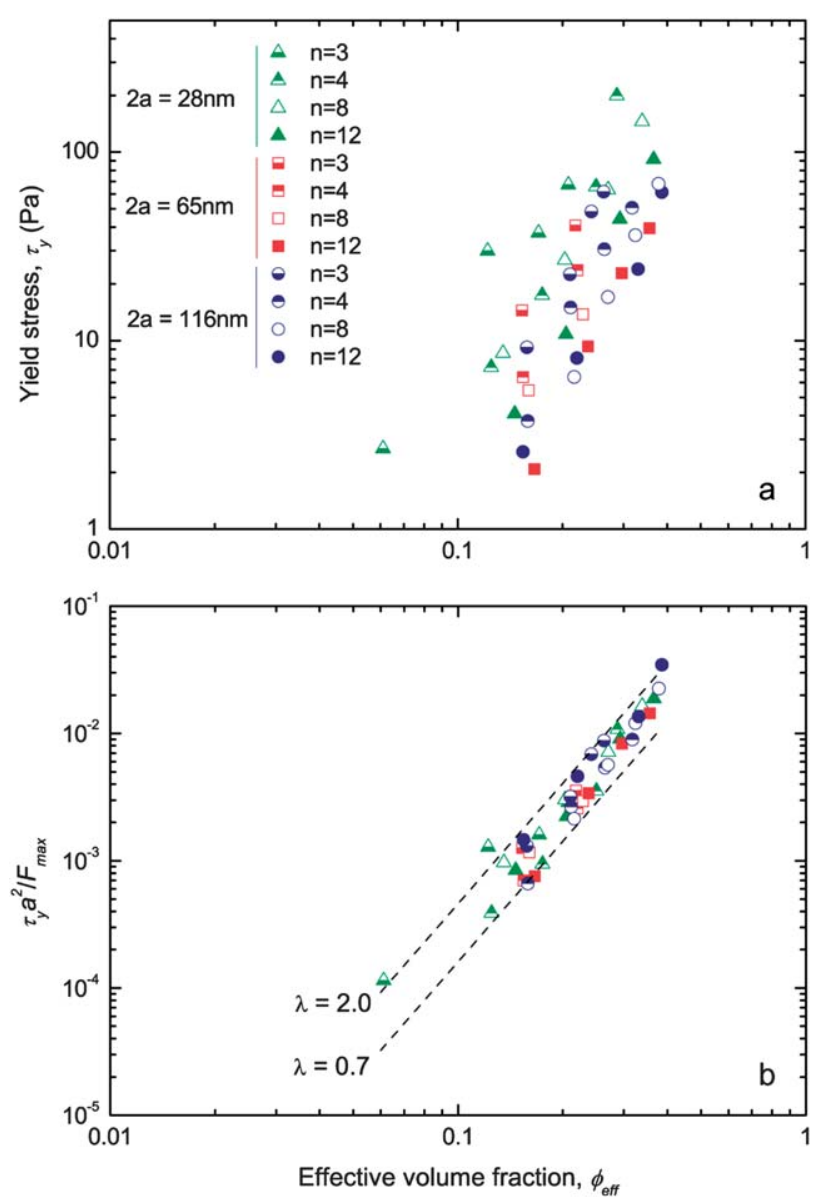

Fig. 4 (a) Yield stress $\left(\tau_{\mathrm{y}}\right)$ as a function of the effective volume fraction of particles $\left(\phi_{\text {eff }}\right)$ obtained for weakly attractive networks containing particles of different sizes $(2 a)$ coated with surface ligands with variable chain lengths $(n)$. (b) Scaling obtained after normalization of the yield stress data. The dashed lines are values expected from eqn (2) for $d_{\mathrm{f}}=$ 2.36 and two arbitrary values of $\lambda=\sum_{i=1}^{j} \cos \theta_{i}$.

a wide range of $F_{\max }, a$ and $\phi_{\text {eff }}$ values (Fig. 4a) collapse into a much narrower range after the proposed normalization of the yield stress (Fig. 4b).

The slope of a linear fit to all the experimental data shown in Fig. $4 \mathrm{~b}$ is taken as the power exponent $\gamma$ (eqn (1)). A $\gamma$ value of 3.14 is obtained, which lies within the typical range of 1.4 to 5.5 observed for this parameter in previous studies. ${ }^{10-14,19-26}$

From the $\gamma$ value obtained, we estimate a fractal dimension $d_{\mathrm{f}}=$ 2.36 for the aggregates that form the colloidal network. This fractal dimension is in good agreement with the range 2.1-2.4 expected for aggregates in networks containing volume fractions of particles varying from 0.10 to $0.40 .^{30-32}$

For a power exponent $\gamma$ of 3.14, we estimate the upper and lower limits of the term $\sum_{i=1}^{j} \cos \theta_{i}$ in eqn (2) required to describe the range of normalized yield stress data shown in Fig. 4b. Remarkably, more than $95 \%$ of the experimental data can be described by our theoretical prediction (eqn (2)) if the term $\sum_{i=1}^{j} \cos \theta_{i}$ is assumed to vary within a relatively narrow range between 0.7 and 2.0. While further studies are required to experimentally quantify $j$ and $\theta$, these lower and upper limits for the factor $\sum_{i=1}^{j} \cos \theta_{i}$ provide insights into the yielding mechanism of such attractive networks.

For a hypothetically large number of critical bonds, e.g. $j=4$, all the four bonding angles $\theta_{i}$ have to lie within the narrow range $60-90^{\circ}$ in order to satisfy the experimental upper limit of $\sum_{i=1}^{j} \cos \theta_{i}=2$. Such allowable range of bonding angles becomes even narrower for $j>4$. Since the probability of finding aggregates with such narrow distribution of bonding angles within the network is low, it is likely that the yielding process is governed by the rupture of only a few (i.e. 1-3) critical interparticle bonds within each aggregate. Thus, our results suggest that the range of $\sum_{i=1}^{j} \cos \theta_{i}$ values obtained from the experimental data is not accidental but directly reflects the statistical distribution of $j$ and $\theta_{i}$ values of the aggregates of the attractive particle networks. A quantitative analysis of the distribution of bonding angles and number of critical bonds of aggregates within attractive networks should be carried out in future studies to fully validate this finding.

In conclusion, we propose a scaling law that explicitly correlates the yield stress of weakly attractive networks with the maximum attractive force between particles, the particle size and the effective volume fraction of particles. The proposed relation shows good agreement with our experimental data, if the number of critical interparticle bonds within the network aggregates are assumed to vary within a relatively narrow range. Analysis of the upper and lower limits of this range suggests that macroscopic yielding in weakly attractive networks is ultimately caused by the rupture of a few interparticle bonds within the aggregates of the load-bearing gel. Future work should address the applicability of this simple relation to other colloidal systems, including a more detailed investigation and statistical description of the actual number of critical bonds $j$ and bonding angles $\theta_{i}$ in such attractive particle networks.

\section{Acknowledgements}

We thank the Swiss National Science Foundation for the financial support (Grant No. 200021-100570/1), and Prof. David Weitz, Prof. Chanjoong Kim, Prof. Emanuela Del Gado and Dr Marco Lattuada for fruitful discussions.

\section{References}

1 J. E. Smay, J. Cesarano and J. A. Lewis, Langmuir, 2002, 18, 54295437.

2 R. Mezzenga, P. Schurtenberger, A. Burbidge and M. Michel, Nat. Mater., 2005, 4, 729-740.

3 N. C. Schirmer, C. Kullmann, M. S. Schmid, B. R. Burg, T. Schwamb and D. Poulikakos, Adv. Mater., 2010, 22, 4701.

4 A. R. Studart, E. Amstad and L. J. Gauckler, Langmuir, 2007, 23, 1081-1090.

5 I. Schenker, F. T. Filser, T. Aste and L. J. Gauckler, J. Eur. Ceram. Soc., 2008, 28, 1443-1449.

6 C. F. Goodeve, Trans. Faraday Soc., 1939, 35, 342-358.

7 R. J. Hunter and S. K. Nicol, J. Colloid Interface Sci., 1968, 28, 250.

8 W. Y. Shih, W. H. Shih and I. A. Aksay, J. Am. Ceram. Soc., 1999, 82, 616-624.

9 Y. K. Leong, P. J. Scales, T. W. Healy, D. V. Boger and R. Buscall, J. Chem. Soc., Faraday Trans., 1993, 89, 2473-2478. 
10 A. A. Potanin, R. Derooij, D. Vandenende and J. Mellema, J. Chem. Phys., 1995, 102, 5845-5853.

11 G. M. Channell and C. F. Zukoski, AIChE J., 1997, 43, 1700-1708.

12 T. F. Tadros, Adv. Colloid Interface Sci., 1996, 68, 97-200.

13 Y. K. Leong, P. J. Scales, T. W. Healy and D. V. Boger, J. Am. Ceram. Soc., 1995, 78, 2209-2212.

14 Y. Zhou, H. Yu, E. J. Wanless, G. J. Jameson and G. V. Franks, J. Colloid Interface Sci., 2009, 336, 533-543.

15 T. Gillespie, J. Colloid Sci., 1960, 15, 219-231.

16 W. B. Russel, D. A. Saville and W. R. Schowalter, Colloidal Dispersions, Cambridge University Press, Cambridge, UK, 1989.

17 R. Derooij, A. A. Potanin, D. Vandenende and J. Mellema, Colloid J., 1994, 56, 476-486.

18 B. A. Firth and R. J. Hunter, J. Colloid Interface Sci., 1976, 57, 248256.

19 D. G. Thomas, AIChE J., 1961, 7, 431-437.

20 R. Buscall, I. J. McGowan, P. D. A. Mills, R. F. Stewart, D. Sutton, L. R. White and G. E. Yates, J. Non-Newtonian Fluid Mech., 1987, 24, 183-202.

21 R. Buscall, P. D. A. Mills, J. W. Goodwin and D. W. Lawson, J. Chem. Soc., Faraday Trans. 1, 1988, 84, 4249-4260.
22 D. Megias-Alguacil, J. D. G. Duran and A. V. Delgado, J. Colloid Interface Sci., 2000, 231, 74-83.

23 E. Vanderaerschot and J. Mewis, Colloids Surf., 1992, 69, 15-22.

24 R. Derooij, A. A. Potanin, D. Vandenende and J. Mellema, J. Chem. Phys., 1993, 99, 9213-9223.

25 R. Derooij, D. Vandenende, M. H. G. Duits and J. Mellema, Phys. Rev. E: Stat. Phys., Plasmas, Fluids, Relat. Interdiscip. Top., 1994, 49, 3038-3049.

26 J. A. Yanez, T. Shikata, F. E. Lange and D. S. Pearson, J. Am. Ceram. Soc., 1996, 79, 2917-2924.

27 A. H. L. West, J. R. Melrose and R. C. Ball, Phys. Rev. E: Stat. Phys., Plasmas, Fluids, Relat. Interdiscip. Top., 1994, 49, 4237-4249.

28 E. M. Furst and J. P. Pantina, Phys. Rev. E: Stat., Nonlinear, Soft Matter Phys., 2007, 75, 050402.

29 A. R. Studart, E. Amstad, M. Antoni and L. J. Gauckler, J. Am. Ceram. Soc., 2006, 89, 2418-2425.

30 M. Lattuada, H. Wu, A. Hasmy and M. Morbidelli, Langmuir, 2003, 19, 6312-6316.

31 A. E. Gonzalez, M. Lach-Hab and E. Blaisten-Barojas, J. Sol-Gel Sci. Technol., 1999, 15, 119-127.

32 A. E. Gonzalez, F. Martinez-Lopez, A. Moncho-Jorda and R. Hidalgo-Alvarez, Phys. A, 2002, 314, 235-245. 